\title{
Synthesis of Nano-Pomegranate Peel via Sol-Gel Method and Screen Characterization
}

\author{
${ }^{1}$ Awatif I. Muhammed $*$, ${ }^{2}$ Quraish A. Kazem, ${ }^{1}$ Rana A. Kamal, ${ }^{1}$ Ahmed J. Issa, ${ }^{1}$ Inas F. Abed, ${ }^{1}$ Salma Ali, \\ ${ }^{3}$ Maan Ali, ${ }^{4}$ Safaa Ali \\ ${ }^{1}$ Veterinary Drug Research and Production Center/ Corporation of Research and Industrial Development - Iraq \\ ${ }^{2}$ Chemical and Petrochemical Research Center/ Corporation of Research and Industrial Development - Iraq \\ ${ }^{3}$ National Center for Packing and Packaging/ Corporation of Research and Industrial Development - Iraq \\ ${ }^{4}$ Al-Razi Center for Research and Medical Kits Production/ Corporation of Research and Industrial Development \\ - Iraq
}

\section{Article history: \\ Keywords: \\ Pomegranate peel, \\ SPM, \\ SEM, \\ Antibacterial test}

Article information

Received: October, 01, 2021

Accepted: November, 12, 2021

Available online: December, 14, 2021

*Corresponding Author:

Awatif I. Muhammed

muhammedawatif@gmail.com

DOI:

https://doi.org/10.53523/ijoirVol8I3ID92

\begin{abstract}
Nano peel solution was prepared using Sol-gel technology at a temperature of (80) Celsius. A number of tests were performed to describe the properties and structure of Nano material, including scanning probe microscopy (SPM), which showed the symmetric cumulative distribution of the solution Nanoparticles and the average grain size equal to $64.5 \mathrm{~nm}$ and the identical distribution of the Nanoparticles with a diameter of 37.75 nanometers, with a measurement area ranging between (1531.23-1558.19) nanometers. Nano-solution analyzed with a scanning electron microscope (SEM), Inspect type (S50), with a magnification power up to X2000, where dense flakes of nano particles with a diameter of $(50 \pm 10)$ nanometers were observed. The antibacterial activity of nano-solution by using gram- positive bacteria St. aurous and gram-negative E. coli show the inhibition diameter of (St. aurous) was $27 \mathrm{~mm}$ and $25 \mathrm{~mm}$ in (E. coli).
\end{abstract}

\section{Introduction}

Study of Nanomaterial is recently widespread due to their large space of Sol-gel used [1]. Nanoparticles made in Sol-gel application considered safe theoretically and when conjugated with herbal extract may potentially activate the activity of herb besides producing nano herbal extract nontoxic and valuable in the natural system [2]. Nano herbal extract is distinguished from others by its their small size, high surface-to-volume ratio, electronic properties, functional groups and aggregation behavior, high efficiency, and ability to penetrate the tissues of different organs as a good source of drug for the treatment of many diseases [3, 4]. Pomegranate peel considers as a rich source of polyphenols with potent antioxidant properties [5] and antibacterial properties. Peels are widely used to treat infections found in human sexual organs, in addition, to treat mastitis, acne, folliculitis, pile, allergic dermatitis, scalds, diarrhea, and dysentery [6,7]. So the presences study concerns with the preparation of Nanomaterial by Sol-gel methods and characterization. 


\section{Experimental Procedure}

\section{Preparation of Nano-Pomegranate Peel Solution}

$100 \mathrm{~g}$ of pomegranate peel collected washed with tap water then exposed the peels to sunlight for two days after derided grinding by a coffee grinder to a fine powder. 1 gram of crusts powder is taken and dissolved with 100 $\mathrm{ml}$ quantity of (ethanol alcohol, water, and acetic acid) placed in a glass beaker of 1 liter capacity and stirred using Ultrasonic Probe sonicator type (USA) and add to the Beaker $10 \mathrm{ml}$ of concentrated acetic acid with the addition of $100 \mathrm{ml}$ of distilled water and by continuous stirring at a temperature of $80^{\circ} \mathrm{C}$ for 4 hours for 10 days.

\section{Characterization of Nano Pomegranate Peel Solution}

\section{Scanning Probe Microscope (SPM) and Scanning Electron Microscope (SEM)}

Scanning Probe Microscopy technique used to measure mechanical, magnetic, electrical, and electrochemical surface topography on a nanometer scale. It can measure features from as small as interatomic spaces to a tenth of a millimeter. The ability to measure surfaces in three dimensions provides more precise topographical data of air and liquids beside the analysis the size of particles in nano-solution [8].

\section{Antibacterial Activity of Nano Solution}

The diffusion method was used to evaluate antimicrobial activity by using of Mueller-Hilton agar medium inoculated with bacterial suspension then incubated at $35{ }^{\circ} \mathrm{C}$ for $24 \mathrm{~h}$., finally inhibition zones were measured considered as antibacterial activity [9].

\section{Results and Discussion}

Figure (1) shows the homogenous distribution of nanoparticles in peel solution and Table (1) shows the average diameter of nanoparticles is $64.5 \mathrm{~nm}$.
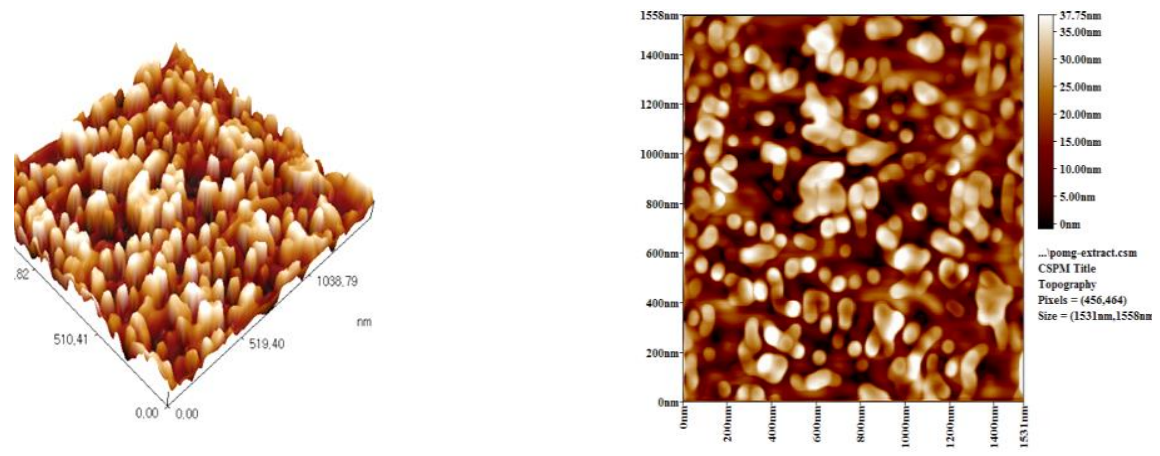

Figure (1). Homogenous distribution of particle by SPM.

Table (1). Diameter of Nano pomegranate peel solution by SPM.

\begin{tabular}{|c|}
\hline $10 \%$ of Nano-particles at $40 \mathrm{~nm}$ \\
\hline $50 \%$ of Nano-particles at $60 \mathrm{~nm}$ \\
\hline $90 \%$ of Nano-particles at $85 \mathrm{~nm}$ \\
\hline Avg. Diameter of Nanoparticles $64.5 \mathrm{~nm}$ \\
\hline
\end{tabular}

The Nanoparticles of peel solution were observed as fine sheets with diameter rate (64.5) $\mathrm{nm}$ scanning by electron microscope (SEM) as seen in Figure (2). 

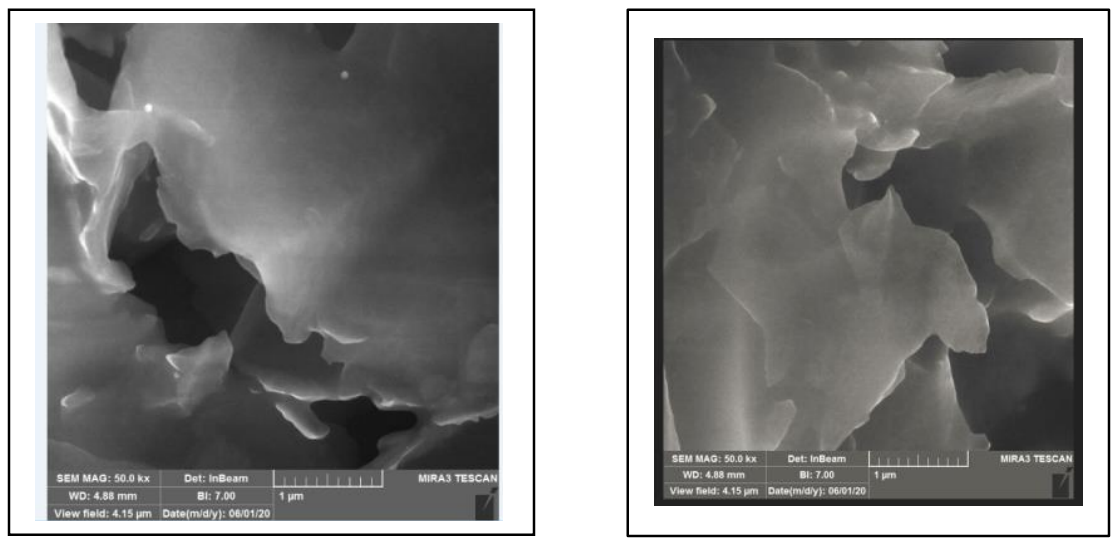

Figure (2). Nano peel analyzed by SEM show fine sheets.

EDS pattern of Nanoparticles was shown in Figure (3) which observe two peaks of carbon and oxygen which acted as a capping agent to nanoparticles and in Table (2) shows the element present in solution.

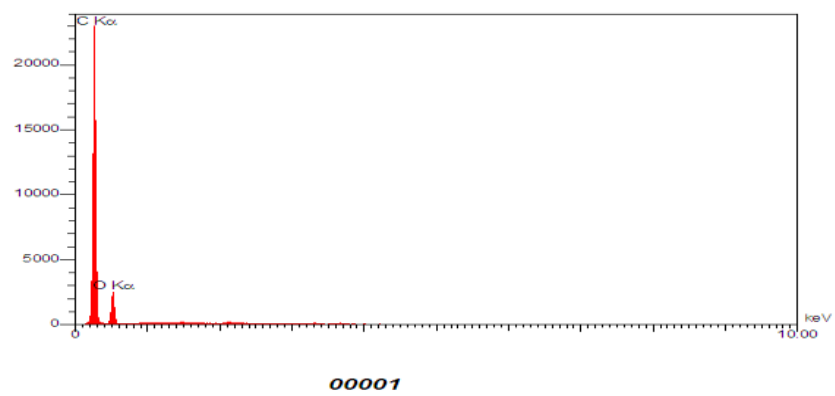

Figure (3). EDX pattern of pomegranate peel Nanoparticles by FE-SEM.

Table (2). Pomegranate peel Nanoparticles elements components.

\begin{tabular}{llllllll|lllll}
\hline $\begin{array}{l}\text { El } \\
\mathrm{t}\end{array}$ & Line & Error & $\mathrm{K}$ & $\mathrm{Kr}$ & $\mathrm{W} \%$ & $\mathrm{~A} \%$ & $\mathrm{ZAF}$ & $\begin{array}{l}\text { Ox } \\
\%\end{array}$ & $\mathrm{Pk} / \mathrm{Bg}$ & $\begin{array}{c}\text { Cla } \\
\mathrm{ss}\end{array}$ & LConf & HConf \\
\hline $\mathbf{C}$ & $\mathrm{Ka}$ & 148.364 & 0.8830 & 0.4861 & 67.21 & 73.19 & 0.7231 & 0.00 & 6030.22 & $\mathrm{~A}$ & 66.66 & 67.76 \\
& & 3 & & & & & & & & & & \\
$\mathbf{O}$ & $\mathrm{Ka}$ & 40.8585 & 0.1170 & 0.0644 & 32.79 & 26.81 & 0.1965 & 0.00 & 760.55 & $\mathrm{~A}$ & 32.05 & 33.53 \\
& & & 1.0000 & 0.5505 & 100.00 & 100.00 & & 0.00 & & & & \\
\hline
\end{tabular}
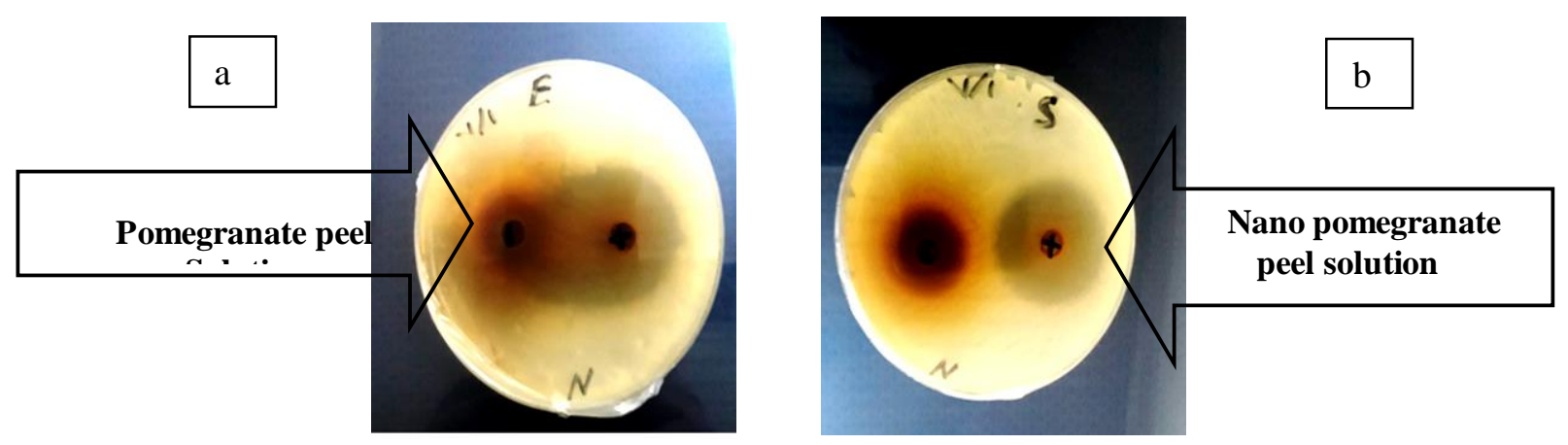

Figure (4). Antibacterial activity of pomegranate peels solution in gram-positive and negative bacteria. 
The antibacterial effect of Nano pomegranate peel solution shows good antimicrobial activity against Escherichia coli and Staphylococcus aureus according to the solution. The inhibition diameter of Nano peel solution in St. aurous was $25 \mathrm{~mm}$ and in E.coli was $27 \mathrm{~mm}$ while crude solution shows $(20,15) \mathrm{mm}$.

\section{Conclusion}

In the study, Nano pomegranate peels solution prepared by Sol-gel method which is considered one of the simplest methods of preparation by using temperature $80^{\circ} \mathrm{C}$. The Nano solution analyzed by AFM shows (64.5) nm diameter range and EDX image show two peaks, the high peak representing carbon at $67.2 \%$ at w\% and low peak representing oxygen at $32.7 \%$ at $w \%$. The antibacterial activity of Nano solution shows diameter between (25-27) $\mathrm{mm}$ in St. aurous and E.coli compared to crude solution due to elements presence made it more efficient and can be used in different fields of pharmaceutical industry in the future.

\section{Reference}

[1] B.Debjit, G.Harish, K.Pragati, S.Duraivel, G.Aravind and K.Sampath "Medicinal Uses of Punica granatum and Its Health Benefits", Pharmacognosy and Phytochemistry journal, vol.1, p. 1-28, 2013.

[2] H. R. Arshad, A. A. Mohamed, A. A. Saleh "Active Constituents of Pomegranates (Punica granatum) as Potential Candidates in the Management of Health through Modulation of Biological Activities", Pharmacogn J., vol.9, p. 689-695, 2017.

[3] E. M, Altuner "Investigation of antimicrobial activity of Punica granatum L. fruit peel ash used for protection against skin infections as folk remedies especially after male circumcision", African Microbiolial J. Research, vol. 5, p. 3339-3342, 2015.

[4] M. Hajoori, M. Naik, K. Naik, and S. Desair" Evaluation of antimicrobial activity of Punica granatum peel extracts using different solvent system", International Pharmacology Screen Method J., vol. 4, p. 26-31, 2014.

[5] M. Nazhan, A. Hamady, T. Mohammed" Green synthesis of silver Nano particles by using (Rheum) extract and its anti-bacterial activity", University of Anbar for pure science Journal, vol.10 , p. 1991-8941, 2016.

[6] B. Rachida, Y. Imene, B. Rachida, B. Sarah, R. Ali'Phytochemical analysis, antibacterial and antioxidant activities of pomegranate (Punica granatum L.) peel extracts", International Journal of Biosciences ,vol.16,p. 35-44, 2020.

[7] G.Marslin, K.Siram, Q.Maqbool et al. "Secondary metabolites in the green synthesis of metallic nanoparticles", Materials Journal, vol. 11, p. 1-940, 2018.

[8] Z .Naziri, H .Rajaian, R.Firouzi”Antibacterial effects of Iranian native sour and sweet pomegranate (Punica granatum) peel extracts against various pathogenic bacteria" Iranian Journal of Veterinary Research, vol. 13,p. 282-288, 2012.

[9] R.R Mphahlele, O.A Fawole, U.L Opara"Effect of drying on the bioactive compounds antioxidant , antibacterial and antityrosinase activities of pomegranate peel", BMC Complementary Medecine and Therapy Journal, vol. 16, p.1-12, 2016. 